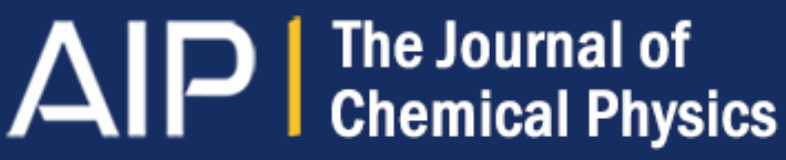

Spin-rotation and NMR shielding constants in $\mathrm{HCl}$

Micha Jaszuski, Michal Repisky, Taye B. Demissie, Stanislav Komorovsky, Elena Malkin, Kenneth Ruud, Piotr Garbacz, Karol Jackowski, and Wodzimierz Makulski

Citation: The Journal of Chemical Physics 139, 234302 (2013); doi: 10.1063/1.4840295

View online: http://dx.doi.org/10.1063/1.4840295

View Table of Contents: http://scitation.aip.org/content/aip/journal/jcp/139/23?ver=pdfcov

Published by the AIP Publishing

\section{$\underset{\substack{\text { Aublosing } \\ \text { sils }}}{A}$ Re-register for Table of Content Alerts}

\section{Create a profile.




\title{
Spin-rotation and NMR shielding constants in $\mathrm{HCl}$
}

\author{
Michał Jaszuński, ${ }^{1, \text { a) }}$ Michal Repisky, ${ }^{2}$ Taye B. Demissie, ${ }^{2, b)}$ Stanislav Komorovsky, ${ }^{2}$ \\ Elena Malkin, ${ }^{2}$ Kenneth Ruud, ${ }^{2}$ Piotr Garbacz, ${ }^{3}$ Karol Jackowski, ${ }^{3}$ \\ and Włodzimierz Makulski ${ }^{3}$ \\ ${ }^{1}$ Institute of Organic Chemistry, Polish Academy of Sciences, 01-224 Warszawa, Kasprzaka 44, Poland \\ ${ }^{2}$ Centre for Theoretical and Computational Chemistry, University of Troms $\phi$-The Arctic University of \\ Norway, N-9037 Troms $\phi$, Norway \\ ${ }^{3}$ Laboratory of NMR Spectroscopy, Department of Chemistry, University of Warsaw, Pasteura 1, 02-093 \\ Warsaw, Poland
}

(Received 9 August 2013; accepted 21 November 2013; published online 16 December 2013)

\begin{abstract}
The spin-rotation and nuclear magnetic shielding constants are analysed for both nuclei in the $\mathrm{HCl}$ molecule. Nonrelativistic ab initio calculations at the $\operatorname{CSSD}(\mathrm{T})$ level of approximation show that it is essential to include relativistic effects to obtain spin-rotation constants consistent with accurate experimental data. Our best estimates for the spin-rotation constants of ${ }^{1} \mathrm{H}^{35} \mathrm{Cl}$ are $C_{\mathrm{Cl}}=-53.914 \mathrm{kHz}$ and $C_{\mathrm{H}}=42.672 \mathrm{kHz}$ (for the lowest rovibrational level). For the chlorine shielding constant, the $a b$ initio value computed including the relativistic corrections, $\sigma(\mathrm{Cl})=976.202 \mathrm{ppm}$, provides a new absolute shielding scale; for hydrogen we find $\sigma(\mathrm{H})=31.403 \mathrm{ppm}$ (both at $300 \mathrm{~K}$ ). Combining the theoretical results with our new gas-phase NMR experimental data allows us to improve the accuracy of the magnetic dipole moments of both chlorine isotopes. For the hydrogen shielding constant, including relativistic effects yields better agreement between experimental and computed values. (C) 2013 AIP Publishing LLC. [http://dx.doi.org/10.1063/1.4840295]
\end{abstract}

\section{INTRODUCTION}

The interaction of nuclear magnetic dipole moments with a magnetic field supplies valuable information about molecular structure. These interactions are the reason for the existence of the Nuclear Magnetic Resonance (NMR) spectra. In a molecule, the direct Zeeman interactions of the applied external magnetic field with the nuclear dipole moments are modified by the electron density. The shielding of each nucleus depends on the molecular electronic structure, and the differences in the local magnetic field are reflected in the chemical shifts observed in NMR spectroscopy. The rotational spectrum is also affected by the nuclear magnetic moments; when they interact with an effective magnetic field arising from the molecular rotation, there is an additional splitting of the bands in the spectrum.

In $a b$ initio studies of molecular properties, these effects are described using perturbation theory. ${ }^{1,2}$ The relevant spectroscopic parameters correspond to second-order molecular properties, which in the perturbation expansion of the molecular energy describe the terms bilinear in the nuclear magnetic moment and either the magnetic field strength or the rotational magnetic moment of the molecule. The computed parameters - NMR shielding constants and spin-rotation constants can be used to predict and interpret the NMR and rotational spectra, respectively.

The comparison of computed spin-rotation constants with experiment is straightforward. In contrast, the shielding constant describes, by definition, the shielding of a bare

\footnotetext{
a)E-mail: michal.jaszunski@icho.edu.pl.

b) Permanent address: Institute of Physical Chemistry, Polish Academy of Sciences, Kasprzaka 44/52 Warsaw, Poland.
}

nucleus by all the electrons in a molecule, whereas the usually measured chemical shift describes the difference between the shielding of a nucleus in the molecule of interest and in a reference molecule. Thus, although calculated and experimental chemical shifts can be compared, to obtain absolute shielding constants from experiment requires a different approach.

The computed shielding constant consists of two contributions, the so-called diamagnetic and paramagnetic terms. The former corresponds to an expectation value, and once a relatively accurate unperturbed wavefunction is available, it is usually easy to determine. However, the calculation of the paramagnetic contribution requires the solution of linear response equations, and therefore this term is much more complicated to calculate accurately. It was shown by Ramsey $^{3}$ (for linear molecules) and by Flygare ${ }^{4,5}$ that the paramagnetic contribution to the NMR shielding constant in the nonrelativistic framework (computed with the gauge origin at the position of that nucleus) is proportional to the electronic part of the spin-rotation constant. Therefore, an approach that has been successfully applied to determine absolute shielding constants (see, e.g., Ref. 6) is to add the calculated diamagnetic term to the paramagnetic term extracted from the experimentally measured spin-rotation constant.

However, the proportionality relation proposed by Ramsey $^{3}$ and Flygare ${ }^{4,5}$ is valid only in nonrelativistic perturbation theory, where both the paramagnetic contribution to the NMR shielding constant $\left(\sigma^{p}\right)$ and the electronic part of the spin-rotation constant $\left(\mathbf{C}^{e l}\right)$ are represented by the angular momentum operator $\vec{L}$. In four-component relativistic theory, $\sigma^{p}$ is represented by the relativistic electronic magnetic 
moment operator $\vec{\mu}_{e}$

$$
\vec{\mu}_{e}=-\frac{1}{2}\left[\vec{r} \times\left(\begin{array}{cc}
\mathbf{0} & \vec{\sigma} \\
\overrightarrow{\boldsymbol{\sigma}} & \mathbf{0}
\end{array}\right)\right],
$$

where $\vec{\sigma}$ is the vector composed of Pauli spin matrices, whereas $\mathbf{C}^{e l}$ is represented by the electronic total angular momentum operator ${ }^{7}$

$$
\overrightarrow{\mathbf{J}}_{e}=\vec{L} \mathbf{1}_{4 \times 4}+\frac{1}{2}\left(\begin{array}{cc}
\vec{\sigma} & \mathbf{0} \\
\mathbf{0} & \overrightarrow{\boldsymbol{\sigma}}
\end{array}\right) .
$$

Further theoretical details can be found in Refs.7-9. Note that $\overrightarrow{\boldsymbol{\mu}}_{e}$ is an off-diagonal operator and therefore couples the large and the small components of the four-component wavefunction, whereas $\overrightarrow{\mathbf{J}}_{e}$ is a purely diagonal operator. This different coupling leads to a change in the physical mechanism of the interactions and gives rise to relativistic corrections that are different for NMR shielding constants and spin-rotation constants. Although the different mechanisms were already discussed by Saue and co-workers, ${ }^{10,11}$ its quantitative estimate was not known until very recently. ${ }^{12,13}$ Surprisingly, the difference has a sizable effect on experimentally determined absolute shielding scales, obtained indirectly through measurements of nuclear spin-rotation constants, showing that the use of Flygare's relation may be inadequate and lead to an incorrect absolute shielding scale. For instance, Malkin et al. ${ }^{12}$ predicted a correction of about $1000 \mathrm{ppm}$ to ${ }^{119} \mathrm{Sn}$ shielding constants of ${ }^{119} \mathrm{SnX}_{4}\left(\mathrm{X}=\mathrm{H}, \mathrm{Cl}, \mathrm{CH}_{3}\right)$. Similarly, Aucar et al. ${ }^{13}$ demonstrated this breakdown in the $\mathrm{HBr}$ and $\mathrm{HI}$ molecules. In this work we examine the role of different effects in the determination of spectroscopic parameters for the $\mathrm{HCl}$ molecule, focusing on the breakdown of Flygare's relation in the relativistic domain.

\section{AB INITIO CALCULATIONS}

\section{A. Nonrelativistic approach}

All the nonrelativistic calculations were performed using the coupled-cluster analytic linear response methods developed by Gauss and Stanton ${ }^{14,15}$ and implemented in the CFOUR program. ${ }^{16}$ All the properties were computed at the $\operatorname{CCSD}(\mathrm{T})$ (coupled-cluster singles-and-doubles with a noniterative perturbative triples correction) level of approximation, for the equilibrium geometry we have in addition estimated the results at the CCSDT level.

We have used a sequence of uncontracted aug-ccpCVXZ basis sets and applied gauge-including atomic orbitals (GIAOs ${ }^{17,18}$ ). The largest basis set in this sequence, the uncontracted aug-cc-pCV5Z basis set, ${ }^{19}$ includes $21 \mathrm{~s} 13 \mathrm{p} 9 \mathrm{~d} 7 \mathrm{f} 5 \mathrm{~g} 3 \mathrm{~h}$ functions for the $\mathrm{Cl}$ atom and $9 \mathrm{~s} 5 \mathrm{p} 4 \mathrm{~d} 3 \mathrm{f} 2 \mathrm{~g}$ for the $\mathrm{H}$ atom, leading to a total of 315 GTO's. In addition, at the equilibrium geometry $\mathrm{r}_{e}=1.2738767 \AA$ (optimised at the $\operatorname{CCSD}(\mathrm{T})$ level with the uncontracted aug-cc-pCV5Z basis set) we have also estimated the results by applying basis set extrapolations. We have applied two-point extrapolations, separately for the Hartree-Fock (HF) and for the correlation contribution to the shielding constants, in each case following Ref. 20. Finally, we have also used for comparison the augpcS- $4^{21}$ basis set. As shown in Table I, the uncontracted aug-
TABLE I. Correlation and basis set dependence of the equilibrium geometry

\begin{tabular}{|c|c|c|c|c|}
\hline & $C_{\mathrm{Cl}}$ & $C_{\mathrm{H}}$ & $\sigma(\mathrm{Cl})$ & $\sigma(\mathrm{H})$ \\
\hline \multicolumn{5}{|l|}{ aug-cc-pCV5Z ${ }^{\mathrm{a}}$ results } \\
\hline $\mathrm{HF}$ & -54.653 & 39.789 & 951.199 & 30.464 \\
\hline CCSD & -51.891 & 40.164 & 961.265 & 30.689 \\
\hline $\operatorname{CCSD}(\mathrm{T})$ & -51.464 & 40.341 & 962.744 & 30.730 \\
\hline \multicolumn{5}{|c|}{ CCSDT - CCSD(T), aug-cc-pCVTZ results } \\
\hline full triples ${ }^{\mathrm{a}}$ & -0.234 & 0.038 & -0.824 & 0.018 \\
\hline full triples ${ }^{\mathrm{b}}$ & -0.234 & 0.038 & -0.820 & 0.018 \\
\hline \multicolumn{5}{|l|}{$\operatorname{CCSD}(\mathrm{T})$ results } \\
\hline aug-cc-pCVTZ ${ }^{\text {a }}$ & -51.316 & 41.289 & 963.056 & 31.057 \\
\hline aug-cc-pCVQZ & -51.456 & 40.595 & 962.710 & 30.816 \\
\hline aug-cc-pCV5Z ${ }^{a}$ & -51.464 & 40.341 & 962.744 & 30.730 \\
\hline \multicolumn{5}{|l|}{ extrapolated results ${ }^{\mathrm{c}}$} \\
\hline TZ-QZ & -51.321 & 40.220 & 963.281 & 30.671 \\
\hline QZ-5Z & -51.437 & 40.175 & 962.903 & 30.669 \\
\hline $\operatorname{CCSD}(\mathrm{T})$, aug-pcS-4 & -51.570 & 40.322 & 962.254 & 30.731 \\
\hline
\end{tabular}
values; $C_{\mathrm{Cl}}$ and $C_{\mathrm{H}}$ in $\mathrm{kHz}, \sigma(\mathrm{Cl})$ and $\sigma(\mathrm{H})$ in ppm.

${ }^{a}$ Uncontracted aug-cc-pCVXZ basis set.

${ }^{\mathrm{b}}$ Contracted aug-cc-pCVTZ basis set.

${ }^{\mathrm{c} T Z-Q Z}$ and QZ-5Z: extrapolation according to Ref. 20, see the text.

cc-pCV5Z, extrapolated and aug-pcS-4 results do not differ significantly. Our nonrelativistic, equilibrium geometry values of $\sigma(\mathrm{Cl})$ are also in good agreement with another recent $\operatorname{CCSD}(\mathrm{T})$ result extrapolated to the complete basis limit, $959.7 \mathrm{ppm}^{22}$

Additionally, we have estimated, using the uncontracted aug-cc-pCVTZ basis set, the CCSDT-CCSD(T) difference, and we will in our discussion refer to this as the "full triples" correction. For all properties, the values of this correction obtained with a smaller, contracted aug-ccpCVTZ basis set, are practically identical to the values we report.

The rovibrational effects have been evaluated at the $\operatorname{CCSD}(\mathrm{T})$ level with the uncontracted aug-cc-pCV5Z basis set. To compare the theoretical and experimental data we need the values for the lowest rovibrational level for the spinrotation constants, and the values at $300 \mathrm{~K}$ for the shielding constants. The corresponding results, that is, the zeropoint vibrational (ZPV) correction and the temperature effect, are given in Table II. The calculated total effect for $\sigma\left({ }^{35} \mathrm{Cl}\right)$ is slightly larger than an earlier estimate, $-15.9 \mathrm{ppm} .{ }^{23} \mathrm{We}$ have determined also the rovibrational corrections for ${ }^{1} \mathrm{H}^{37} \mathrm{Cl}$. However, since the computed differences between the shielding constants of ${ }^{35} \mathrm{Cl}$ and ${ }^{37} \mathrm{Cl}(\approx 0.1 \mathrm{ppm}$ at $300 \mathrm{~K})$ are far smaller than the residual errors in the calculations, we do not analyse these differences.

\section{B. Relativistic effects}

The relativistic expressions defining the spin-rotation constants were not known until very recently. In 2012 Aucar et $\mathrm{al}^{7}$ proposed a theory where the nuclei are considered as nonrelativisic particles while the electrons are treated relativistically. More recently, Xiao and $\mathrm{Liu}^{9}$ (see also references therein) developed a theory where the nuclei are 
TABLE II. A comparison of ab initio and experimental results; $C_{\mathrm{Cl}}$ and $C_{\mathrm{H}}$ in $\mathrm{kHz}, \sigma(\mathrm{Cl})$ and $\sigma(\mathrm{H})$ in ppm.

\begin{tabular}{|c|c|c|c|c|}
\hline & $C_{\mathrm{Cl}}$ & $C_{\mathrm{H}}$ & $\sigma(\mathrm{Cl})$ & $\sigma(\mathrm{H})$ \\
\hline \multicolumn{5}{|c|}{ Equilibrium geometry values } \\
\hline \multicolumn{5}{|l|}{$\operatorname{CCSD}(\mathrm{T})$} \\
\hline $\begin{array}{l}\text { aug-cc-pCV5Z } \\
\text { Full triples }\end{array}$ & $\begin{array}{r}-51.464 \\
-0.234\end{array}$ & $\begin{array}{r}40.341 \\
0.038\end{array}$ & $\begin{array}{r}962.744 \\
-0.824\end{array}$ & $\begin{array}{r}30.730 \\
0.018\end{array}$ \\
\hline Relativistic ${ }^{c}$ & -0.026 & 2.548 & 31.819 & 0.841 \\
\hline \multicolumn{5}{|c|}{ Rovibrational effects ${ }^{\mathrm{d}}$} \\
\hline ZPV & -2.223 & -0.425 & -17.087 & -0.263 \\
\hline $300 \mathrm{~K}$ & $\ldots$ & $\ldots$ & -0.591 & -0.023 \\
\hline Total $^{\mathrm{e}}$ & \multicolumn{2}{|c|}{$\mathrm{v}=0$} & \multicolumn{2}{|c|}{$300 \mathrm{~K}$} \\
\hline & -53.914 & 42.672 & 976.202 & 31.403 \\
\hline Expt. ${ }^{\mathrm{f}}$ & $54.00(15)$ & $-42.32(70)$ & $(-)^{\mathrm{g}}$ & $31.124(7)$ \\
\hline
\end{tabular}

${ }^{a}$ Uncontracted aug-cc-pCV5Z basis set.

${ }^{\mathrm{b}}$ Full triples correction: CCSDT-CCSD(T), see the text and Table I.

${ }^{\mathrm{c}}$ Relativistic correction: PBE, uncontracted pc-3 basis set, see the text.

${ }^{\mathrm{d}}$ Nonrelativistic results, ${ }^{35} \mathrm{Cl}$ and ${ }^{1} \mathrm{H}$.

${ }^{\mathrm{e}}$ For both properties, rovibrational corrections to the relativistic contribution are included: $0.033 \mathrm{kHz}, 0.170 \mathrm{kHz}, 0.141 \mathrm{ppm}$, and $0.101 \mathrm{ppm}$, respectively.

${ }^{\text {f}}$ Spin-rotation constants: Ref. 53 (different sign convention used); $\sigma(\mathrm{H})$ : Ref. 48 (31.132 ppm in this work).

${ }^{\mathrm{g}}$ We assume that for $\sigma(\mathrm{Cl})$ our $a b$ initio value sets the absolute shielding scale.

described quasi-relativistically and the electrons relativistically; the same authors next described the theory for the special case of linear molecules. ${ }^{24}$ Our implementation follows the work of Aucar et al. ${ }^{7}$ and its details will be published in Ref. 8.

To estimate the relativistic effects on the NMR shielding constants and spin-rotation constants, we have used the fourcomponent relativistic density functional theory (DFT) program package ReSpect, ${ }^{25}$ in particular modules for the calculation of NMR shielding constants ${ }^{26,27}$ and nuclear spinrotation constants. ${ }^{8}$ The relativistic effects (see Table II) were estimated as the differences between four-component values obtained at the DFT level with the PBE functional ${ }^{28}$ and the corresponding nonrelativistic values. We have verified that the relativistic corrections computed with the PBE and BP86 ${ }^{29,30}$ functionals are very similar for both nuclei and both properties. For numerical integration, an adaptive grid with 19784 grid points was used. At both levels, relativistic and nonrelativistic, we used the uncontracted pc-3 ([17s13p4d2f1g] Cl and [9s4p2d1f] H) basis set. ${ }^{31,32}$ Similar results have been obtained with a smaller uncontracted pc-2 basis set. The calculations of NMR shielding constants were performed using GIAOs, while in the calculations of nuclear spin-rotation constants the gauge origin was placed at the center of nuclear mass. The following nuclear magnetic moments were used: $2.79284734 \mu_{\mathrm{N}}$ for ${ }^{1} \mathrm{H}$ and $0.8218743 \mu_{\mathrm{N}}$ for ${ }^{35} \mathrm{Cl} .{ }^{33}$

The relativistic DFT corrections to the spin-rotation constants calculated in the present work $\left(2.548 \mathrm{kHz}\right.$ for ${ }^{1} \mathrm{H}$ and $-0.026 \mathrm{kHz}$ for ${ }^{35} \mathrm{Cl}$ ) are in good agreement with earlier estimates obtained at the Hartree-Fock level of theory, $2.12 \mathrm{kHz}$ for ${ }^{1} \mathrm{H}$ and $-0.04 \mathrm{kHz}$ for ${ }^{35} \mathrm{Cl} .{ }^{13}$

The relativistic theory for the calculation of NMR shielding tensors has been known much longer and numerous calculations of the relativistic effects on the shielding constants have been published (for recent and comprehensive reviews see, e.g., Refs.34-36; in particular more than 25 results obtained for the $\mathrm{HCl}$ molecule are discussed in Ref.34). Our results are in good agreement with those obtained by Manninen et al. ${ }^{37}$ at the Hartree-Fock level of theory; the relativistic corrections estimated using Breit-Pauli perturbation theory were $30.27 \mathrm{ppm}$ for chlorine and $1.144 \mathrm{ppm}$ for the hydrogen shielding constants, respectively. The total $\sigma(\mathrm{Cl})$ derived by adding the relativistic correction to the HF result, $983.0 \mathrm{ppm}$, is also in close agreement with a recent DKH2HF value, $984.1 \mathrm{ppm}^{38}$ Our best estimate for the total equilibrium geometry value of the chlorine shielding constant, 993.739 ppm, is in good agreement with the result derived applying the experimental spin-rotation constant and the relativistic mapping between the properties as discussed above, 995.61 ppm. $^{39}$

\section{GAS PHASE NMR EXPERIMENT}

All the experimental results reported in this paper were obtained in the gas phase. NMR samples were prepared according to our standard procedure described in Ref. 40. We used pure hydrogen chloride $(\geq 99 \% \mathrm{HCl}$, AldrichSigma) with the natural abundance of chlorine isotopes and helium-3 ( $\geq 99.95 \%{ }^{3} \mathrm{He}$, Isotec) without further purification. Gas samples in cylindrical $4 \mathrm{~mm}$ o.d. ampoules contained $\mathrm{HCl}$ (pressure range $\approx 10-40$ bars) with a small quantity of helium-3 ( $\approx 65$ mbar). Liquid benzene- $\mathrm{d}_{6}\left(99.96 \% \quad \mathrm{C}_{6} \mathrm{D}_{6}\right.$, Aldrich-Sigma) was applied for the external deuterium lock. The ${ }^{1} \mathrm{H},{ }^{35} \mathrm{Cl}$, and ${ }^{37} \mathrm{Cl}$ frequencies were observed using the BB-10 probe on a Varian INOVA-500 NMR spectrometer with the following parameters: pulse $\pi / 2(\approx 30 \mu \mathrm{s})$, acquisition time $2 \mathrm{~s}\left({ }^{1} \mathrm{H}\right)$ or $25 \mathrm{~ms}\left({ }^{35} \mathrm{Cl}\right.$ and $\left.{ }^{37} \mathrm{Cl}\right)$, scan numbers approximately 100 for ${ }^{1} \mathrm{H}$ and 10000 for ${ }^{35} \mathrm{Cl}$ or ${ }^{37} \mathrm{Cl}$. The ${ }^{3} \mathrm{He} \mathrm{NMR}$ experiments were performed as described earlier. ${ }^{41}$ The external magnetic field was stable, $\mathrm{B}_{0}=11.758 \mathrm{~T}$.

The results of our experimental study, performed in a constant magnetic field, are summarized by the plots in Fig. 1. As shown, the decrease of the ${ }^{3} \mathrm{He}$ resonance frequency with $\mathrm{HCl}$ density is significant, and it comes almost exclusively from the bulk susceptibility correction (BSC). ${ }^{42}$ In contrast, the density dependence of the ${ }^{35} \mathrm{Cl}$ and ${ }^{37} \mathrm{Cl}$ frequencies are in the opposite direction showing the significant effects due to intermolecular interactions. This means that the intermolecular effects in ${ }^{35} \mathrm{Cl}$ and ${ }^{37} \mathrm{Cl} \mathrm{NMR}$ are much stronger than the BSC parameter itself; the latter factor is exactly the same for all the studied nuclei in the same sample. Finally, the ${ }^{1} \mathrm{H}$ frequency dependence on the density is almost negligible, indicating that in this case the effects of the intermolecular interactions are roughly balanced by BSC. The latter result is slightly different from the first observation of $\mathrm{HCl}$ in the gas phase, done by Raynes et al. in 1962, ${ }^{43}$ where a stronger intermolecular effect in the proton shielding than BSC in our experiment was found (in a constant external magnetic field the resonance frequencies are directly proportional to the nuclear magnetic shielding, justifying these comments on intermolecular effects in proton shielding). Fig. 1 also presents the primary ${ }^{35} \mathrm{Cl} /{ }^{37} \mathrm{Cl}$ isotope effect in the resonance frequency 


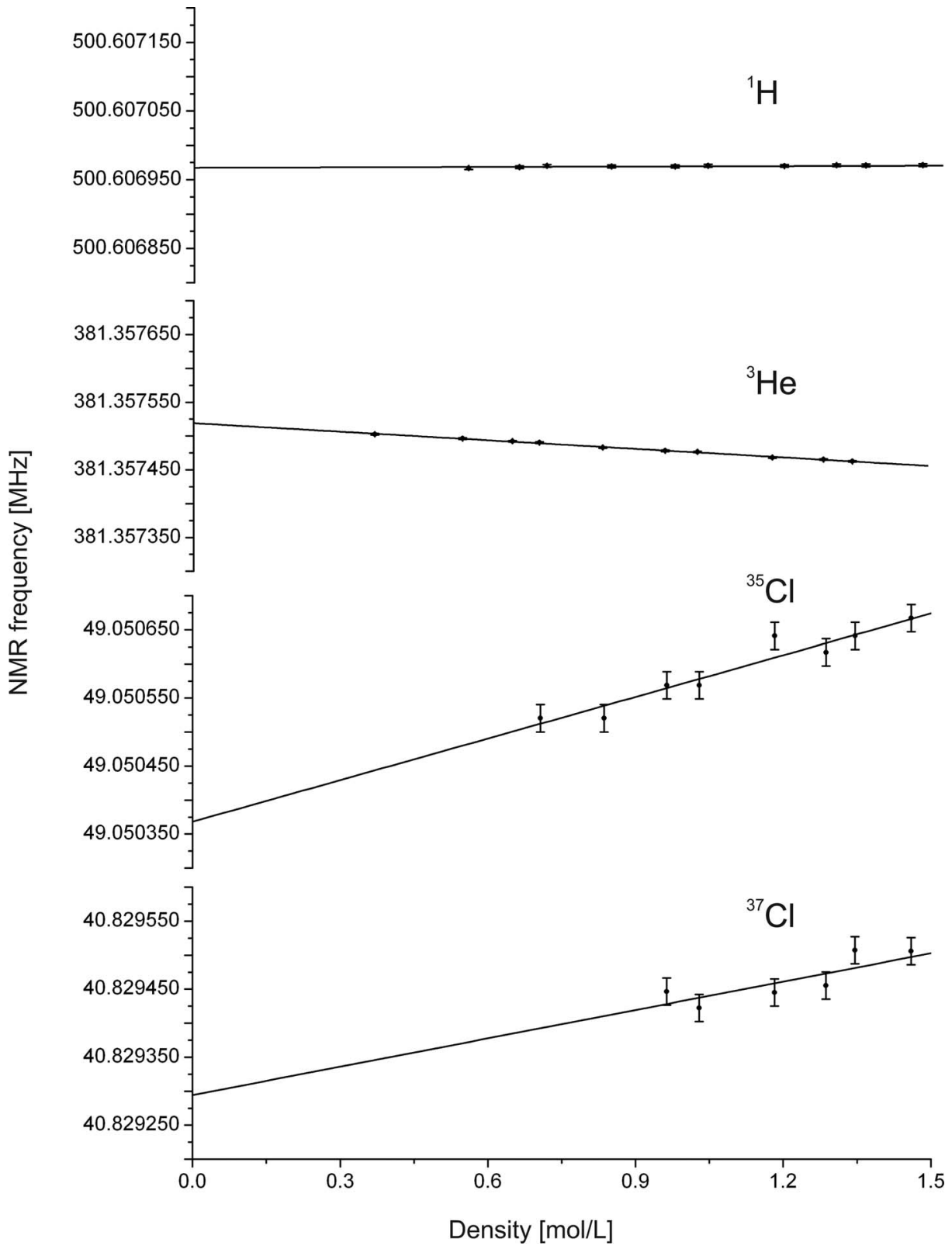

FIG. 1. Density dependence of ${ }^{1} \mathrm{H},{ }^{3} \mathrm{He},{ }^{35} \mathrm{Cl}$, and ${ }^{37} \mathrm{Cl}$ resonance frequencies measured for gaseous $\mathrm{HCl}$ samples containing a small amount of helium-3 (see Sec. III for details).

for an isolated $\mathrm{HCl}$ molecule. The observations of pure isotope effects are possible only for gaseous compounds and deliver important data for theoretical considerations, as has already been shown for the deuterium isotopomers of methyl fluoride. ${ }^{44}$

For comparison with the ab initio values, computed for an isolated molecule, we need the experimental values at the zero-density limit. Linear extrapolation of the experimental data points to this limit gives: $v\left({ }^{1} \mathrm{H}\right)=500.6069675(13)$, $v\left({ }^{3} \mathrm{He}\right)=381.3575191(7), v\left({ }^{35} \mathrm{Cl}\right)=49.050368(20)$, and $v\left({ }^{37} \mathrm{Cl}\right)=40.829294(42) \mathrm{MHz}$. The error bars are larger for ${ }^{35} \mathrm{Cl}$ and ${ }^{37} \mathrm{Cl}$ than for ${ }^{1} \mathrm{H}$ and ${ }^{3} \mathrm{He}$, reflecting the non-zero electric quadrupole moment of both chlorine isotopes; the case of ${ }^{37} \mathrm{Cl}$ is particularly difficult due to the low natural abundance of this isotope (24.47\%). In the comparison of ex- perimental and theoretical values we shall therefore focus on the results for ${ }^{1} \mathrm{H}^{35} \mathrm{Cl}$.

\section{NMR SHIELDING CONSTANTS}

The shielding constant of a nucleus $\mathrm{X}, \sigma_{\mathrm{X}}$, can be determined from the experimental data as

$$
\sigma_{X}=1-\frac{v_{X}}{v_{Y}} \frac{\Delta \mu_{Y}^{z}}{\Delta \mu_{X}^{z}}\left(1-\sigma_{Y}\right)
$$

where $v_{Z}, \mu_{Z}$, and $I_{Z}$ are the resonance frequencies, the nuclear magnetic moments and the spin numbers of both nuclei $(\mathrm{Z}=\mathrm{X}, \mathrm{Y})$ and $\Delta \mu_{\mathrm{Z}}^{z}=\mu_{\mathrm{Z}} \Delta I_{\mathrm{Z}}^{z} / I_{\mathrm{Z}}$ is the increment of the projection of the magnetic moment on the axis of the external field. This equation is easily derived considering the 
resonance frequencies of two different nuclei, $v_{\mathrm{X}}$ and $v_{\mathrm{Y}}$,

$$
\begin{aligned}
& h v_{\mathrm{X}}=\Delta \mu_{\mathrm{X}}^{z}\left(1-\sigma_{\mathrm{X}}\right) B_{0}, \\
& h v_{\mathrm{Y}}=\Delta \mu_{\mathrm{Y}}^{z}\left(1-\sigma_{\mathrm{Y}}\right) B_{0},
\end{aligned}
$$

and eliminating the external magnetic field induction $B_{0}$. We shall assume here that all the necessary projection, sign and spin-related coefficients can be easily taken care of and analyse the values of $\mu_{\mathrm{Z}}$. We stress that Eq. (3) may be used only when the magnetic moments of both nuclei are known with satisfactory accuracy. ${ }^{45}$

This procedure can certainly be applied to evaluate $\sigma\left({ }^{1} \mathrm{H}\right)$ in $\mathrm{HCl}$, because very accurate magnetic moments of ${ }^{1} \mathrm{H}$ and ${ }^{3} \mathrm{He}$ are known. We use $\mu\left({ }^{1} \mathrm{H}\right)=2.792847356(23) \mu_{\mathrm{N}}$ and $\mu\left({ }^{3} \mathrm{He}\right)=2.127625306(25) \mu_{\mathrm{N}}$, determined from $\sigma\left({ }^{3} \mathrm{He}\right)$ $=59.96743(10) \mathrm{ppm}^{46}$ (see Refs. 41 and $47, \mu_{\mathrm{N}}$ is the nuclear magneton). The result, $\sigma\left({ }^{1} \mathrm{H}\right)=31.132 \mathrm{ppm}$, is in very good agreement with another recent experimental value, 31.124(7) ppm. ${ }^{48}$

\section{A. Nuclear magnetic dipole moments of ${ }^{35} \mathrm{Cl}$ and ${ }^{37} \mathrm{Cl}$}

When NMR shielding constants are known with higher accuracy than one of the nuclear magnetic dipole moments, Eqs. (4) and (5) may be applied in a different manner. Namely, one can determine this unknown magnetic moment $\mu_{\mathrm{X}}$ as

$$
\Delta \mu_{\mathrm{X}}^{z}=\frac{v_{\mathrm{X}}}{v_{\mathrm{Y}}} \frac{\left(1-\sigma_{\mathrm{Y}}\right)}{\left(1-\sigma_{\mathrm{X}}\right)} \Delta \mu_{\mathrm{Y}}^{z} .
$$

We consider this to be the case for the magnetic dipole moments of the ${ }^{35} \mathrm{Cl}$ and ${ }^{37} \mathrm{Cl}$ isotopes and use this equation to evaluate their accurate values. These moments have been recently estimated to be $0.821698(13)$ and $0.683977(11) \mu_{N}$, respectively. ${ }^{49}$ Both values are somewhat smaller than the older IUPAC literature data, $0.8218743(4) \mu_{\mathrm{N}}$ for ${ }^{35} \mathrm{Cl}$ and $0.6841236(4) \mu_{\mathrm{N}}$ for ${ }^{37} \mathrm{Cl} .{ }^{33}$ However, to obtain this estimate, a nonrelativistic value of the shielding of $\mathrm{Cl}^{-}$ion in water, taken from Ref. 23, has been used. Not surprisingly, when our nonrelativistic values for the chlorine shielding constants in gaseous $\mathrm{HCl}$ are taken as input data we obtain very similar results: $0.821695 \mu_{\mathrm{N}}$ and $0.683975 \mu_{\mathrm{N}}$, respectively.

Undoubtedly, more reliable values can be derived using in Eq. (6) for the chlorine shielding constants the total values which include also the relativistic corrections. We can compare the shielding constant $\sigma_{\mathrm{X}}$ and resonance frequency $v_{\mathrm{X}}$ of the nuclide of interest with the parameters of a proton $\left(\mu_{\mathrm{H}}, \sigma_{\mathrm{H}}, v_{\mathrm{H}}\right)$ in the same isolated molecule. ${ }^{50,51}$ In this approach (that is, $\mathrm{X}=\mathrm{Cl}, \mathrm{Y}=\mathrm{H}$ in Eq. (6)) and applying the experimental hydrogen shielding value $31.124 \mathrm{ppm}$ as input we find $0.821721(5) \mu_{\mathrm{N}}$ for ${ }^{35} \mathrm{Cl}$ and $0.683997(4) \mu_{\mathrm{N}}$ for ${ }^{37} \mathrm{Cl}$ (to estimate the error bars, we assumed the accuracy of the shielding constants to be $\pm 5 \mathrm{ppm}$ for chlorine and $\pm 0.5 \mathrm{ppm}$ for hydrogen ${ }^{49}$ ). We can also determine the magnetic moments considering helium-3 as our reference, and from Eq. (6) with $\mathrm{X}=\mathrm{Cl}, \mathrm{Y}=\mathrm{He}$ we obtain practically identical results. As expected, a noticeable change in the evaluated nuclear magnetic dipole moments is observed when we take into account the relativistic contribution to the chlorine shielding constants.

\section{CONCLUSIONS}

For numerous nuclei the absolute shielding scales have been established analysing the spin-rotation constants in small molecules and applying Flygare's relation to determine the paramagnetic contribution. Although it was known that this relation is valid only in a nonrelativistic approach, ${ }^{10,11}$ computed $a b$ initio shielding constants were usually not sufficiently accurate to provide a useful alternative approach. More recently, the development of accurate nonrelativistic methods and - in particular — the progress in the calculation of spin-rotation and shielding constants at the relativistic level of theory enabled the analysis of the role of Flygare's approximation. ${ }^{12,13}$ It has been previously shown that using the spin-rotation data for tin compounds leads to very large errors in NMR shielding constant; for much lighter elements such as chlorine, the discrepancies should be much smaller.

Nevertheless, we find that the accuracy of the results for $\mathrm{HCl}$ is significantly improved when we take into account relativistic effects. For the chlorine spin-rotation constant, the relativistic correction is practically negligible, but for hydrogen it is very significant, being about $5 \%$ of the final value. The effect of relativity is significant, not only when the desired properties are calculated directly for the heavy centers, but also when they are calculated for a lighter atom in the vicinity of a heavy one (heavy-atom effect on the shielding of the light atom, HALA ${ }^{52}$ ). This can be traced to the observation that the spin-orbit interaction, which is the most important contributor to the HALA effect on NMR shielding constants, also is an important contribution to the spin-rotation constants. ${ }^{7,8}$ Even though we estimate the corrections from a relativistic DFT approach, the agreement with experiment is clearly improved compared to the nonrelativistic value. Also for the hydrogen shielding constant, adding the relativistic correction undoubtedly improves the agreement with experiment. For chlorine, our estimate of the (large) relativistic effect on the shielding is in agreement with other $a b$ initio results. We have also measured the chemical shift of ${ }^{35} \mathrm{Cl}$ in gaseous $\mathrm{HCl}$ with respect to the solvated chlorine ion. It is $29.76 \mathrm{ppm}$, thus we finally estimate the absolute shielding constant of the standard NMR chlorine reference $\left(0.1 \mathrm{M}\right.$ solution of $\mathrm{NaCl}$ in $\left.\mathrm{D}_{2} \mathrm{O}\right)$ as 1006 $\pm 5 \mathrm{ppm}$. The change with respect to the value given in Ref. 23 (974 \pm 4 ppm, obtained with a measured $28.5 \mathrm{ppm}$ chemical shift) is almost entirely due to the relativistic correction, which we have now included for $\mathrm{HCl}$. The differences between the ${ }^{35} \mathrm{Cl}$ and ${ }^{37} \mathrm{Cl}$ results, $<1 \mathrm{ppm}$, are too small to enable a reliable analysis. We thus assume that previous experimental values, based on a nonrelativistic absolute shielding scale, are less accurate than our ab initio result and suggest using the result presented here to define the absolute scale for the chlorine shielding. This also enables us to determine new values for the chlorine magnetic dipole moments for both isotopes: $0.821721(5) \mu_{\mathrm{N}}$ for ${ }^{35} \mathrm{Cl}$ and $0.683997(4) \mu_{\mathrm{N}}$ for ${ }^{37} \mathrm{Cl}$. For both isotopes, the new values of the nuclear magnetic moments confirm our earlier estimates ${ }^{49}$ and are decreased by $0.02 \%$ with respect to the IUPAC reference data. ${ }^{33}$ The agreement between the measured and calculated hydrogen shielding constant is noticeably improved when we include the large relativistic contribution, $0.841 \mathrm{ppm}$. Finally, 
we recall that the relativistic corrections to the spin-rotation and shielding constants are in this work computed independently, the simple nonrelativistic relation between these two properties does not hold in the relativistic theory. The agreement of the computed properties with the available experimental data confirms the accuracy of the applied approach.

\section{ACKNOWLEDGMENTS}

We are indebted to Dr. Jochen Autschbach and Dr. Wenjian Liu for providing preprints of their works prior to publication. We acknowledge partial support by the National Science Centre (Poland) grant, according to the Decision No. DEC2011/01/B/ST4/06588 (M.J., P.G., K.J., and W.M.). Computational grant from the Norwegian Supercomputing Program (nn4654K) is also gratefully acknowledged. T.B.D. has been supported by the International PhD Projects Programme of the Foundation for Polish Science co-financed from European Regional Development Fund within Innovative Economy Operational Programme Grants for Innovation. We also gratefully acknowledge research grants from the Research Council of Norway: 179568/V30 and 177558/V00 (K.R.), 191251/V30 (E.M.), and 214095-F20 (M.R. and S.K.).

${ }^{1}$ T. Helgaker, M. Jaszuński, and K. Ruud, Chem. Rev. 99, 293 (1999).

${ }^{2}$ T. Helgaker, S. Coriani, P. Jørgensen, K. Kristensen, J. Olsen, and K. Ruud, Chem. Rev. 112, 543 (2012).

${ }^{3}$ N. F. Ramsey, Phys. Rev. 78, 699 (1950).

${ }^{4}$ W. H. Flygare, J. Chem. Phys. 41, 793 (1964).

${ }^{5}$ W. H. Flygare, Chem. Rev. 74, 653 (1974).

${ }^{6}$ D. Sundholm and J. Gauss, Mol. Phys. 92, 1007 (1997).

${ }^{7}$ I. A. Aucar, S. S. Gómez, M. C. Ruiz de Azúa, and C. G. Giribet, J. Chem. Phys. 136, 204119 (2012).

${ }^{8}$ S. Komorovsky, M. Repisky, T. B. Demissie, E. Malkin, and K. Ruud (unpublished).

${ }^{9}$ Y. Xiao and W. Liu, J. Chem. Phys. 138, 134104 (2013).

${ }^{10}$ L. Visscher, T. Enevoldsen, T. Saue, H. J. Aa. Jensen, and J. Oddershede, J. Comput. Chem. 20, 1262 (1999).

${ }^{11}$ T. Saue, Adv. Quantum Chem. 48, 383 (2005).

${ }^{12}$ E. Malkin, S. Komorovsky, M. Repisky, T. B. Demissie, and K. Ruud, J. Phys. Chem. Lett. 4, 459 (2013).

${ }^{13}$ I. A. Aucar, S. S. Gómez, J. I. Melo, C. C. Giribet, and M. C. Ruiz de Azúa, J. Chem. Phys. 138, 134107 (2013).

${ }^{14}$ J. Gauss and J. F. Stanton, J. Chem. Phys. 102, 251 (1995).

${ }^{15}$ J. Gauss and J. F. Stanton, J. Chem. Phys. 104, 2574 (1996).

${ }^{16}$ CFOUR, a quantum chemical program package written by J. F. Stanton, J. Gauss, M. E. Harding, P. G. Szalay with contributions from A. A. Auer, R. J. Bartlett, U. Benedikt, C. Berger, D. E. Bernholdt, O. Christiansen, M. Heckert, O. Heun, C. Huber, D. Jonsson, J. Jusélius, K. Klein, W. J. Lauderdale, D. Matthews, T. Metzroth, D. P. O’Neill, D. R. Price, E. Prochnow, K. Ruud, F. Schiffmann, S. Stopkowicz, A. Tajti, M. E. Varner, J. Vázquez, F. Wang, J. D. Watts and the integral packages MOLECULE (J. Almlöf and P. R. Taylor), PROPS (P. R. Taylor), ABACUS (T. Helgaker, H. J. Aa. Jensen, P. Jørgensen, and J. Olsen), and ECP routines by A. V. Mitin and C. van Wüllen. For the current version, see http://www.cfour.de.

${ }^{17}$ F. London, J. Phys. Radium 8, 397 (1937).

${ }^{18}$ K. Wolinski, J. F. Hinton, and P. Pulay, J. Am. Chem. Soc. 112, 8251 (1990).
${ }^{19}$ K. A. Peterson and T. H. Dunning, Jr., J. Chem. Phys. 117, 10548 (2002).

${ }^{20}$ A. M. Teale, O. B. Lutnæs, T. Helgaker, D. J. Tozer, and J. Gauss, J. Chem. Phys. 138, 024111 (2013).

${ }^{21}$ F. Jensen, J. Chem. Theory Comput. 4, 719 (2008).

${ }^{22}$ M. Sun, I. Y. Zhang, A. Wu, and X. Xu, J. Chem. Phys. 138, 124113 (2013).

${ }^{23}$ M. Gee, R. E. Wasylishen, and A. Laaksonen, J. Phys. Chem. A. 103, 10805 (1999).

${ }^{24}$ Y. Xiao and W. Liu, J. Chem. Phys. 139, 034113 (2013).

${ }^{25}$ ReSpect, version 3.2, 2013; Relativistic Spectroscopy DFT program of authors M. Repisky, S. Komorovsky, V. G. Malkin, O. L. Malkina, M. Kaupp, K. Ruud, with contributions from R. Bast, U. Ekstrom, S. Knecht, I. Malkin Ondik, E. Malkin.

${ }^{26}$ S. Komorovsky, M. Repisky, O. L. Malkina, V. G. Malkin, I. Malkin Ondik, and M. Kaupp, J. Chem. Phys. 128, 104101 (2008).

${ }^{27}$ S. Komorovsky, M. Repisky, O. L. Malkina, and V. G. Malkin, J. Chem. Phys. 132, 154101 (2010).

${ }^{28}$ J. P. Perdew, K. Burke, and M. Ernzerhof, Phys. Rev. Lett. 77, 3865 (1996).

${ }^{29}$ A. D. Becke, Phys. Rev. A 38, 3098 (1988).

${ }^{30}$ J. P. Perdew, Phys. Rev. B 33, 8822 (1986); Erratum: 34, 7406 (1986).

${ }^{31}$ F. Jensen, J. Chem. Phys. 115, 9113 (2001); Erratum: 116, 3502 (2002).

${ }^{32}$ F. Jensen and T. Helgaker, J. Chem. Phys. 121, 3463 (2004).

${ }^{33}$ E. Cohen, T. Cvitaš, J. Frey, B. Holmström, K. Kuchitsu, R. Marquardt, I. Mills, F. Pavese, M. Quack, J. Stohner, H. Strauss, M. Takami, and A. Thor, Quantities, Units and Symbols in Physical Chemistry, IUPAC Green Book, 3rd ed. (IUPAC \& RSC Publishing, Cambridge, 2008).

${ }^{34}$ J. Autschbach and S. Zheng, Annu. Rep. NMR Spectrosc. 67, 1 (2009).

${ }^{35}$ J. Autschbach, J. Chem. Phys. 136, 150902 (2012).

${ }^{36} \mathrm{~J}$. Autschbach, "Relativistic calculations of magnetic resonance parameters: Background and some recent developments," J. Philos. Trans. A (to be published).

${ }^{37}$ P. Manninen, K. Ruud, P. Lantto, and J. Vaara, J. Chem. Phys. 122, 114107 (2005); Erratum, 124, 149901 (2006).

${ }^{38}$ T. Yoshizawa and M. Hada, Chem. Phys. Lett. 580, 145 (2013).

${ }^{39}$ Y. Xiao, Y. Zhang, and W. Liu (unpublished).

${ }^{40}$ K. Jackowski, J. Mol. Struct. 786, 215 (2006).

${ }^{41}$ K. Jackowski, M. Jaszuński, B. Kamieński, and M. Wilczek, J. Magn. Reson. 193, 147 (2008).

${ }^{42}$ P. Garbacz, K. Piszczatowski, K. Jackowski, R. Moszynski, and M. Jaszuński, J. Chem. Phys. 135, 084310 (2011).

${ }^{43}$ W. T. Raynes, A. D. Buckingham, and H. J. Bernstein, J. Chem. Phys. 36, 3481 (1962).

${ }^{44}$ K. Jackowski, M. Kubiszewski, and W. Makulski, J. Mol. Struct. 614, 267 (2002).

${ }^{45}$ M. Jaszuński and K. Jackowski, "Nuclear magnetic dipole moments from NMR spectra - quantum chemistry and experiment," in Lecture Notes in Physics, edited by S. G. Karshenboim (Springer, 2008), Vol. 745, p. 233.

${ }^{46}$ A. Rudziński, M. Puchalski, and K. Pachucki, J. Chem. Phys. 130, 244102 (2009).

${ }^{47}$ P. J. Mohr, B. N. Taylor, and D. B. Newell, Rev. Mod. Phys. 80, 633 (2008).

${ }^{48}$ P. Garbacz, K. Jackowski, W. Makulski, and R. E. Wasylishen, J. Phys. Chem. A 116, 11896 (2012).

${ }^{49}$ M. Jaszuński, A. Antušek, P. Garbacz, K. Jackowski, W. Makulski, and M. Wilczek, Prog. Nucl. Magn. Reson. Spectrosc. 67, 49 (2012).

${ }^{50}$ K. Jackowski and M. Jaszuński, Concepts Magn. Reson., Part A 30, 246 (2007).

${ }^{51}$ A. Antušek, K. Jackowski, M. Jaszuński, W. Makulski, and M. Wilczek, Chem. Phys. Lett. 411, 111 (2005).

${ }^{52}$ P. Pyykkö, A. Görling, and N. Rösch, Mol. Phys. 61, 195 (1987).

${ }^{53}$ G. Cazzoli and C. Puzzarini, J. Mol. Spectrosc. 226, 161 (2004). 